\title{
Temperature Dependent Diquark and Baryon Masses
}

\author{
A. Chandra ${ }^{1}$, A. Bhattacharya ${ }^{1}$, B. Chakrabarti ${ }^{2}$ \\ ${ }^{1}$ Department of Physics, Jadavpur University, Kolkata, India \\ ${ }^{2}$ Department of Physics, Jogamaya Devi College, Kolkata, India \\ Email: pampa@phys.jdvu.ac.in, arpita1chandra@gmail.com, ballari_chakrabarti@yahoo.co.in
}

Received March 21, 2013; revised April 22, 2013; accepted May 19, 2013

Copyright (C) 2013 A. Chandra et al. This is an open access article distributed under the Creative Commons Attribution License, which permits unrestricted use, distribution, and reproduction in any medium, provided the original work is properly cited.

\begin{abstract}
Temperature dependence of diquark mass has been investigated in the frame work of the quasi particle diquark model. The effective mass of the diquark has been suggested to have a temperature dependence which shows a power law behavior. The variation of the diquark mass with temperature has been studied. A decrease in effective mass at temperature $T<T_{c}$, where $T_{c}$ is the critical temperature has been observed. Some features of the phase transition have been discussed. The phase transition is found to be of second order. Temperature variation of baryon masses has also been studied. The results are compared and discussed with available works.
\end{abstract}

Keywords: Diquark; Quasi Particle; Rushbrooke Inequality; Power Law

\section{Introduction}

Quasi particles are particle like entities which arise in some system of interacting particles. The existence of quasi particles is mostly known in condensed matter physics. The quantum phases like superfluidity and superconductivity are largely described by the properties of such low lying excitations which behave like quasi particle simulating many body interactions in the system. An electron in crystal lattice behaves like a quasi particle. It is well known that the behavior of electron and other particles depends upon the environment. The interaction inside can alter the collective properties which produce a new particle called quasi particle. The quantum Hall effect which is an emerging area of research also suggests that the collective behavior of an electron results in a new particle which may have fractional charges but seems to be the constituent of electron. Quasi particles and electrons are same entity in this picture having no distinction as expected between an elementary particle and a quasi particle. The concept of quasi particles has been widely used in describing the system of fermions, superconductivity, superfluidity. Khodel et al. [1] have investigated the properties of fermion system and corresponding phase transition of fermion condensate. With the advent of low temperature experimental technique the quantum critical point phenomena and quantum fluctuation have become area of interest and intense research. Abrahams et al. [2] have investigated critical quasi particle theory and have discussed the scaling behavior asso- ciated with the quantum critical point (QCP). The effect of temperature dependent quasi particle mass which is effective on the surface impedance of a crystal has been studied by Cassinese et al. [3] in the context of two fluid model. Nawa et al. [4] have studied the BEC condensation of composite diquark in the quark matter (color superconductivity) using a quasi chemical theory at a low density in a region near the de-confinement phase transition. They have argued that the dynamical quark-pair fluctuation can be described as bosonic degrees of freedom which are diquarks. Schneider et al. [5] have suggested that at very high temperature the QGP may be considered to be consisted of quasi free quarks and gluons. They have considered a temperature dependent quasi particle effective mass by parametrizing $m^{*}(T) \sim G_{0} T\left[1-\left(T_{c} / T\right)\right]^{\beta}$. Koh [6] has pointed out that the heavy massive quasi particle becomes superconducting in heavy fermion system and has suggested that the phase transition is of second order. The study of hadrons and their masses at finite temperature is extremely important for understanding the phase transition from hadronic phase to QGP phase. It has been suggested that the nucleon mass depends substantially on the temperature variation of quark condensate and relevant interaction process. A number of works have been done on the possible variation of nucleon mass at finite temperature.

In the current work we have studied the temperature dependence of effective mass of diquarks and baryons in 
quasi particle approach. Recently we have proposed a quasi particle model for diquark where diquark has been described as a quasi particle which behaves like low lying excited state resembling the hypothetical phonon particle in quasi quantum systems. We have suggested relation between the temperature and the effective mass of diquark which follows a power law type of behavior. The power law is a useful tool for studying the critical behaviour near the transition point. The quasi particle critical behaviour has been studied by a number of authors. It would be interesting to study how does the mass of the diquark behaves which is also described as a quasi particle in the current work. The baryons are studied in the frame work of diquark-quark system and the variation of masses of baryons with temperature have also been investigated. Critical exponents of different thermodynamic co-ordinates have been studied and they are found to show Rushbrooke inequality indicating a second order phase transition for deconfinement.

\section{Quasi Particle Model of Diquark and Temperature Dependence of Mass}

We have suggested a model [7] in which two quarks are assumed to be correlated to form a low energy configuration, forming a diquark and behaving like a quasi particle in an analogy with an electron behaving as a quasi particle in the crystal lattice [8]. It is well known that a quasi particle is a low-lying excited state whose motion is modified by the interactions within the system. An electron in a crystal is subjected to two types of forces, namely, the effect of the crystal field $(\operatorname{grad} V)$ and an external force $(F)$ which accelerates the electron [8]. Under the influence of these two forces, an electron in a crystal behaves like a quasi particle having velocity $v$ whose effective mass $m^{*}$ reflects the inertia of electrons which are already in a crystal field such that:

$$
m^{*} \frac{\mathrm{d} v}{\mathrm{~d} t}=F
$$

The bare electrons (with normal mass) are affected by the lattice force-grad $V$ (where $V$ is the periodic potential) and the external force $F$ so that:

$$
m \frac{\mathrm{d} v}{\mathrm{~d} x}=F-\frac{\mathrm{d} V}{\mathrm{~d} x}
$$

Hence the ratio of the normal mass $(m)$ to the effective mass $\left(m^{*}\right)$ can be expressed as:

$$
\frac{m}{m^{*}}=1-\frac{1}{F}\left[\frac{\delta V}{\delta x}\right]
$$

An elementary particle in vacuum may be suggested to be in a situation exactly resembling that of an electron in a crystal. We have proposed a similar type of picture for the diquark $[u d]_{0}$ as a quasi particle inside a nucleon.
We assume that the diquark is an independent body which is under the influence of two types of forces. One is due to the background meson cloud which is represented by the potential $V=(-2 / 3)\left(\alpha_{s} / r\right)$, where $\alpha_{s}$ being the strong coupling constant, and this potential resembles the crystal field on a crystal electron. On the other hand for the external force we have considered an average force $F=-a r$, where $a$ is a suitable constant, which is of confinement type. It has been assumed that under the influence of these two types of interactions the diquark is behaving like a quasi particle, a low lying excited state and its mass gets modified. The ratio of the constituent mass and the effective mass of the diquark $\left(m_{D}^{*}\right)$ can be expressed by using the same formalism as in Equation (3) and is obtained as:

$$
\frac{m_{q}+m_{q^{\prime}}}{m_{D}^{*}}=1+\frac{\alpha}{2 a r^{3}}
$$

Here $m_{q}+m_{q}^{\prime}$ represents the normal constituent mass of the diquark and $m_{D}$ is the effective mass of the diquark, $\alpha=(2 / 3) \alpha_{s}, \alpha_{s}=0.58$ [9] and the strength parameter a $=0.003 \mathrm{GeV}^{3}[10]$ for the light and heavy-light diquarks, $V$ being the average value of the one gluon exchange type of potential. " $r$ " is the radius parameter of the diquark. To calculate the effective mass of the diquark from the above expression we need the radius parameter $r$ of the diquark. To calculate the effective mass of the diquark from the above expression we need the radius parameter " $r$ " of the diquark. The radii parameter of the scalar diquarks have been used from existing literature [11-15] and constituent masses of quarks are taken from Karliner et al. [16]. We have estimated the masses of the diquarks in the framework of the quasi particle and the results that obtained are displayed in Table 1. The diquarks which are described as the elementary excitation simulating many body interactions behaves like scalar boson and may be regarded as separate entity. Two diquarks should be antisymmetric in color so that a total color singlet state is obtained. We presume that such a system of low lying excitations behaving like quasi particles does not interact among themselves and as in an ideal gas their energies are additive [17]. Thus the mass of a baryon in a diquark-quark system can be represented as:

$$
M_{B}=m_{D}^{*}+m_{q}
$$

We have parametrized the temperature dependent effective mass of diquark by a power law such that:

$$
m_{D}^{*}(T)=m_{D}^{*}(0)\left(1-\frac{T}{T_{c}}\right)^{-\epsilon}
$$

where " $\epsilon$ " is critical exponent. The critical exponent describes the temperature dependence of the system near 
Table 1. Diquark masses $\left(m^{*}(0)\right)$.

\begin{tabular}{ccc}
\hline \multicolumn{3}{c}{ Diquark } \\
\hline Quark Content & Radius $\left(\mathrm{GeV}^{-1}\right)$ & Mass Computed $(\mathrm{GeV})$ \\
\hline$[\mathrm{ud}]_{0}$ & $5.38[11]$ & 0.509 \\
{$[\mathrm{us}]_{0},[\mathrm{ds}]_{0}$} & $6.06[12]$ & 0.698 \\
{$[\mathrm{ss}]_{0}$} & $3.65[13]$ & 0.985 \\
{$[\mathrm{uc}]_{0},[\mathrm{dc}]_{0}$} & $5.5[14]$ & 1.491 \\
{$[\mathrm{sc}]_{0}$} & $5.4[13]$ & 1.596 \\
{$[\mathrm{sb}]_{0}$} & $4.1[14]$ & 2.887 \\
{$[\mathrm{ub}]_{0},[\mathrm{db}]_{0}$} & $4.4[14]$ & 3.079 \\
{$[\mathrm{cc}]_{0}$} & $2.88[15]$ & 3.287 \\
{$[\mathrm{cb}]_{0}$} & $2.415[15]$ & 6.161 \\
{$[\mathrm{bb}]_{0}$} & $1.75[15]$ & 8.556 \\
\hline
\end{tabular}

the critical point. We have assumed to be $\epsilon=3 / 2$. The mass variation of diquark with temperature has been displayed in Figure 1. We have considered the critical exponent as $3 / 2$ from the concept of statistical model of hadron which is described in details in [18-20]. The probability density of baryon in the ground state is expressed as [18]:

$$
|\psi(r)|^{2}=\frac{315}{64 \pi r_{0}^{9 / 2}}\left(r_{0}-r\right)^{3 / 2} \theta\left(r_{0}-r\right)
$$

where $r_{0}$ is the radius parameter of the corresponding hadron and $\theta\left(r_{0}-r\right)$ represents the step function. It is interesting to note here that the wave function or the probability density possesses a fractional power $3 / 2$ indicating a non analytical behavior $[19,20]$. In the current work we have assumed that the temperature dependence of the diquark mass can be represented by a power law with a critical exponent $3 / 2$ in an analogy with the power law bahaviour of the statistical model wave function as discussed earlier. It would be interesting to investigate how does the above mentioned parameterization yield the mass variation and the thermodynamic properties of the diquarks and the baryons. We define an order parameter by $\eta=\left(1-T / T_{c}\right)^{1 / 2}$ and arrived at:

$$
\eta=\left(1-\frac{T}{T_{c}}\right)^{1 / 2}=t^{1 / 2}=t^{\beta}
$$

where $\beta=1 / 2$ and $\left(1-T / T_{c}\right)=t$. The specific heat $\mathrm{C}$ runs:

$$
C \sim t^{-7 / 2} \sim t^{-\alpha}
$$

The coefficient of volume expansion is obtained as:

$$
\alpha_{T} \sim t^{-1} \sim t^{-\gamma}
$$

We find that $\alpha+2 \beta+\gamma>2$ which satisfy Rushbrooke inequality $\alpha+2 \beta+\gamma \geq 2$ suggesting a second order phase transition for diquark. The variation of effec-

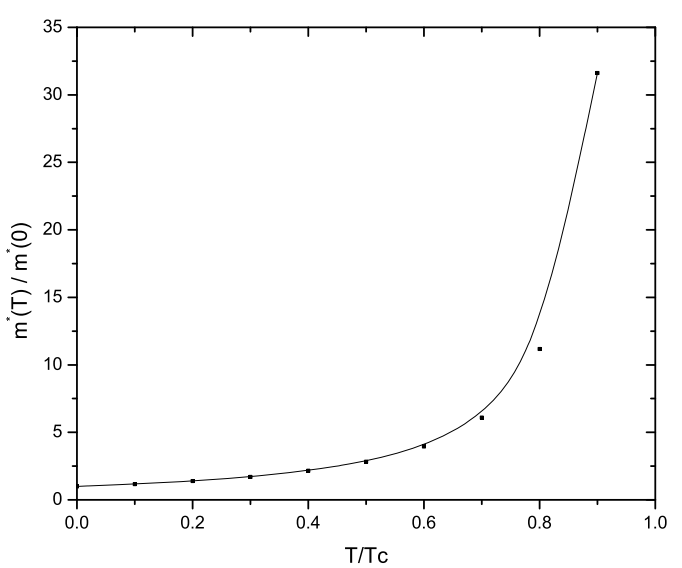

Figure 1. The temperature dependent diquark mass below the critical point.

tive mass with temperature has been estimated for the diquark and the baryons using Equations (5) and (6). The masses of the various baryons in the context of the quasi particle model have been estimated using Equation (5) with temperature dependent diquark mass from Equation (6) with different " $a$ " such as $a=0.04 \mathrm{GeV}^{3}$ for the [ss] diquark (fitting the experimental mass of $\Omega_{c}^{0}$ from PDG2010), $a=0.2 \mathrm{GeV}^{3}$ for doubly heavy diquarks. The results are displayed in Figure 2 for light baryons, Figure 3 for charm sector, Figure 4 for bottom sector and Figure 5 for triply heavy baryons. The masses are found to decrease below $T_{c}$.

\section{Results and Discussions}

In the current work we have investigated the temperature dependent effective mass of diquark incorporating a power law type of behavior with critical exponent $3 / 2$ inspired by the power law behavior of wave function obtained in context of the statistical model [18]. It has been suggested that diquark is a fundamental constituent of hadron and quasi particle in nature formation of which is favoured by the quantum behavior (superfluid) of the vacuum. The mass of diquark has been found to decrease at $T<T_{c}$. The decrease in mass may be attributed to the fact that as the temperature of the system falls below critical value, more and more hypothetical virtual diquarks have been created making the system ordered which changes the interaction of the system. This results in the suppression of the mass enhancement in contrary to the normal state. Koh et al. [6] have pointed out that the quasi particles in heavy fermion system become superconducting with anomalously high mass near transition temperature and the mass decreases with decreasing temperature from $T_{c}$. They have suggested that the phase transition is of second order. Similar observation has been made in the current work. The mass is found to increase anomalously near transition temperature. Results 


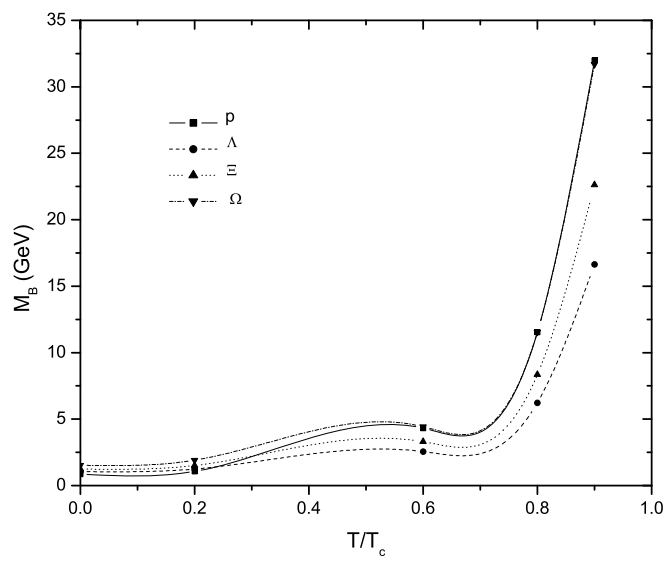

Figure 2. Baryon masses for light-sector as a function of temperature below critical temperature $\left(T=T_{c}\right)$.

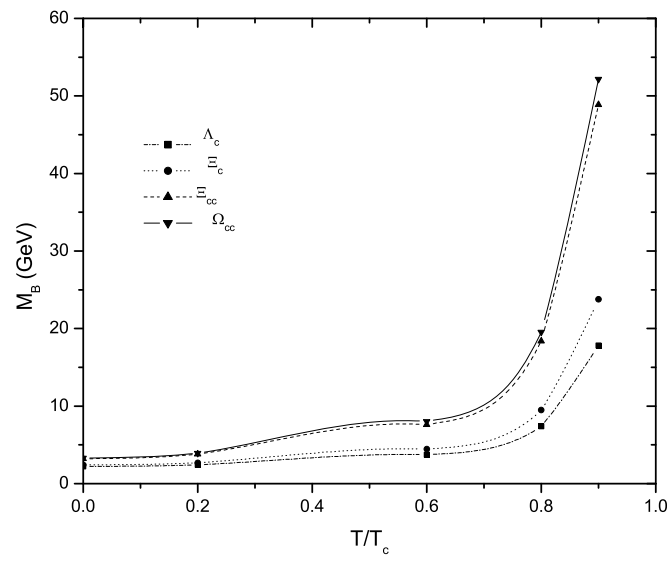

Figure 3. Baryon masses for charm-sector as a function of temperature below critical temperature $\left(T=T_{c}\right)$.

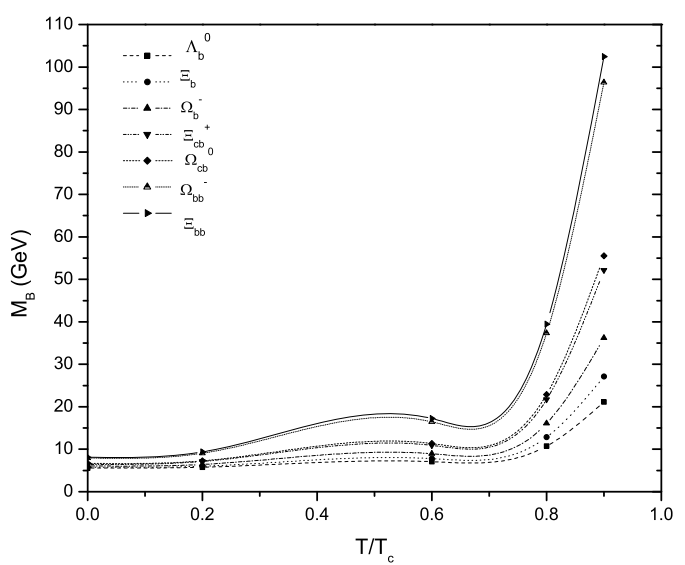

Figure 4. Baryon masses for bottom-sector as a function of temperature below critical temperature $\left(T=T_{c}\right)$.

are displayed in Figures 2-5. The transition from hadronic to deconfinement phase is suggested to be of second order as the dissolution of diquark indicates non existence of baryon in the current scheme. It may be mentioned that temperature dependent hadron masses have

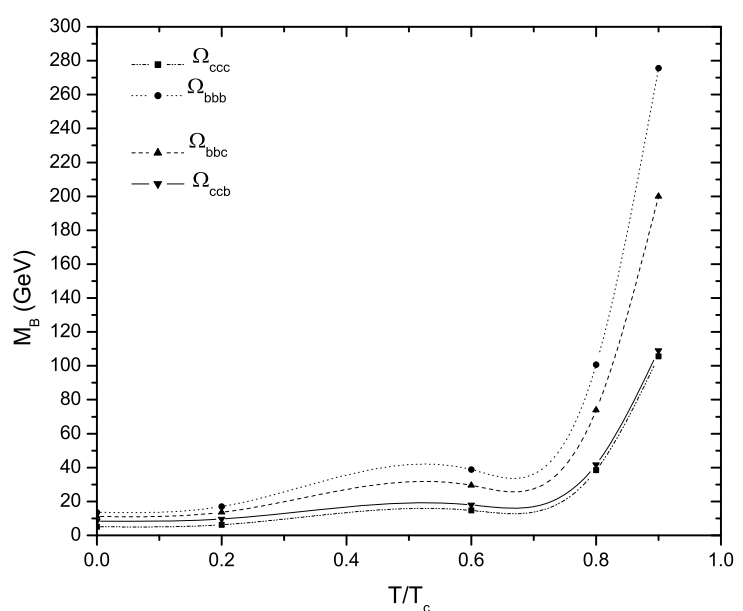

Figure 5. Triply heavy baryon masses as a function of temperature below critical temperature $\left(T=T_{c}\right)$.

been investigated by a number of authors [21-25] in the context of hot dense hadronic matter. Results obtained are divergent in nature and somewhat inconclusive. Some predicts increase in masses with temperature [21,22] whereas some observed just the opposite [23-25]. Eletsky et al. [26] have investigated current correlator in QCD at finite temperature. They have pointed out that the study of current correlator could yield a clear signal for phase transition between QGP at high temperature and hadronic phase at low temperature. At $T<T_{c}$ they have observed a decrease in mass with temperature for $\rho$ and $a_{1}$ meson. The mass shift is found to vary as $\mathrm{T}^{4}$. Zakout [27] has studied nucleon bound state at finite temperature in diquark-quark scheme using Bethe Sal-peter equation with an interaction via exchange quark. The modification of interaction has been approximated by imaginary time formalism in propagator and then an adiabatic approximation. The nucleon mass has been suggested to decrease with temperature as

$M(T)=M(0)\left\{1-b\left(T^{2} / T_{c}^{2}\right)\right\}^{1 / 2}$. It may be mentioned that the critical like divergent equations have been suggested and studied widely in many systems in condensed matter physics [28-30].

\section{Conclusion}

In the current work the temperature variation of baryon masses has been studied considering a power law behavior with critical exponent $3 / 2$ in an analogy with the power law behaviour of the probability density of hadrons obtained in the context of statistical model [19, 20]. The phase transition has been found to be of second order. The power law behavior assigned in the current investigation leads to the results which agree well with the observations made by other authors with quasi particle approach in different context of hadrons and super 
physics. It may be mentioned that the power law behaviors are the manifestation of the dynamics of complex system whose striking feature is the showing of universal laws which are characterized by exponents in scale invariant distribution and they are basically independent of the detailed of the microscopic dynamics [31]. Hadron itself is a complex system. It appears that it may not be far from reality to describe such a complex system by chaos, fractal which shows power law behavior. Further insight in this direction would be done in our future works.

\section{Acknowledgements}

Authors are thankful to University Grants Commission (UGC), New Delhi, India for financial assistance. Ref No. F.No.37-217/2009 (SR).

\section{REFERENCES}

[1] V. A. Khodel and V. R. Shaginyan, Pis'ma Zh. Eksp. Theor. Fiz., Vol. 51, 1990, pp. 488-490.

[2] E. Abrahams, P. Woelfle, Strongly Correlated Electrons, 2012.

[3] A. Cassinese, M. A. Hein, S. Hensen and G. Müller, The European Physical Journal B, Vol. 14, 2000, pp. 605-610. doi: $10.1007 / \mathrm{s} 100510051068$

[4] K. Nawa, et al., Physical Review D, Vol. 74, 2006, Article ID: 034017. doi:10.1103/PhysRevD.74.034017

[5] R. A. Schneider, T. Renk and W. Weise, "Quasiparticles in QCD Thermodynamics and Dilepton Radiation."

[6] S. I. Koh, Physical Review B, Vol. 51, 1995, pp. 669-673. doi:10.1103/PhysRevB.51.11669

[7] A. Bhattacharya, A. Chandra, B. Chakrabarti and A. Sagari, The European Physical Journal Plus, Vol. 126, 2011, pp. 57-61. doi:10.1140/epjp/i2011-11057-1

[8] A. Huang, "Theoretical Solid State Physics," Pergamon Press, Oxford, 1975, p. 100.

[9] W. Lucha, F. F. Schoberi and D. Gromes, Physics Reports, Vol. 200, 1991, pp. 127-240. doi:10.1016/0370-1573(91)90001-3

[10] R. K. Bhaduri, L. E. Cohler and Y. Nogami, Physical Review Letters, Vol. 44, 1980, pp. 1369-1372. doi:10.1103/PhysRevLett.44.1369

[11] B. Chakrabarti, et al., Nuclear Physics A, Vol. 782, 2007, pp. 392-395. doi:10.1016/j.nuclphysa.2006.10.021
[12] A. Bhattacharya, A. sagari, B. Chakrabarti and S. Mani, Physical Review C, Vol. 81, 2010, Article ID: 015202. doi:10.1103/PhysRevC.81.015202

[13] B. Ram and V. Kriss, Physical Review D, Vol. 35, 1987, pp. 400-402. doi:10.1103/PhysRevD.35.400

[14] B. Chakrabarti, Modern Physics Letters A, Vol. 12, 1997, pp. 2133-2140. doi:10.1142/S0217732397002181

[15] A. S. de Castro, H. F. de Carvalho and A. C. B. Antunes, Zeitschrift für Physik C Particles and Fields, Vol. 57, 1993, pp. 315-317. doi:10.1007/BF01565063

[16] M. Karliner and H. J. Lipkin, Physics Letters B, Vol. 575, 2003, pp. 249-255. doi:10.1016/j.physletb.2003.09.062

[17] L. D. Landau and E. M. Lifshitz, "Statistical Physics," Oxford, Vol. 5, 3rd Edition, p. 216.

[18] S. N. Banerjee, A. Bhattacharya, B. Chakrabarti and S. Banerjee, International Journal of Modern Physics A, Vol. 16, 2001, pp. 201-207. doi:10.1142/S0217751X01002956

[19] A. Bhattacharya, A. Raichaudhuri and S. N. Banerjee, Nuovo Cimento, Vol. 105, 1992, pp. 497-501. doi:10.1007/BF02730786

[20] W. W. Kolb, et al., "In the Early Universe," Addison-Wesly Publishing Company, Singapore, p. 485.

[21] J. Gasser and H. Leutwyler, Physics Letters B, Vol. 188, 1987, pp. 477-481. doi:10.1016/0370-2693(87)91652-2

[22] C. M. Shakin and W. D. Sun, Physical Review C, Vol. 49, 1994, pp. 1185-1189. doi:10.1103/PhysRevC.49.1185

[23] C. Song, Physical Review D, Vol. 49, 1994, pp. 15511555. doi:10.1103/PhysRevD.49.1556

[24] K. Saito, T. Maruyama and K. Soutome, Physical Review C, Vol. 40, 1989, pp. 407-431. doi:10.1103/PhysRevC.40.407

[25] T. Hatsuda and T. Kunihiro, Progress of Theoretical Physics, 1987, pp. 284-298. doi:10.1143/PTPS.91.284

[26] V. L. Eletsky and B. L. Ioffe, Physical Review D, Vol. 51, 1995, pp. 2371-2376. doi:10.1103/PhysRevD.51.2371

[27] I. Zakout, Nuclear Theory, 2005, 14 p.

[28] P. T. Renter, et al., "Critical Exponents of Statistical Mal Fragmentation Model," 2001.

[29] H. G. Ballesteros, et al., Physics Letters B, Vol. 387, 1996, p. 125. doi:10.1016/0370-2693(96)00984-7

[30] A. Drozd-Rzoska, et al., Physical Review E, Vol. 82, 2010, Article ID: 031501. doi:10.1103/PhysRevE.82.031501

[31] S. Lehmann, A. D. Jackson and B. Lautrup, Physics and Society, 2005, 5 p. 